\title{
Simultaneidade de comportamentos negativos relacionados à saúde em portadores de diabetes
}

\section{Simultaneity of negative behaviors related to health in people with diabetes}

\author{
Maira Sayuri Sakay Bortoletto; ; Mathias Roberto Loch²; Selma Maffei de \\ Andrade $^{3}$; Maria do Carmo Lourenço Haddad ${ }^{4}$; Alberto Durán González ${ }^{3}$
}

\section{Resumo}

O presente estudo buscou identificar a prevalência isolada e simultânea de comportamentos negativos relacionados à saúde entre indivíduos com diabetes, segundo sexo e classe econômica. Trata-se de estudo transversal, no qual foram entrevistados 337 indivíduos com diabetes em duas unidades básicas de saúde de Londrina, PR. Consideraram-se comportamentos negativos relacionados à saúde o consumo abusivo de álcool, tabagismo, inatividade física no lazer e não realização de dieta. Entre os entrevistados, a inatividade física esteve presente em $69,1 \%$, o consumo abusivo de álcool em $21,1 \%$, a não realização da dieta em $30,6 \%$ e o tabagismo em $10,7 \%$. Diferenças dos comportamentos entre os sexos foram observadas no comportamento inatividade $(\mathrm{p}=0,008)$, maior entre as mulheres, e no tabagismo $(p<0,001)$ e no consumo abusivo de álcool $(p<0,001)$, maior entre os homens. Diferenças entre as classes econômicas e os comportamentos não foram observadas. A simultaneidade de comportamentos relacionados à saúde teve o predomínio da ocorrência da soma de dois comportamentos negativos, e o acúmulo de comportamentos negativos foi mais prevalente entre os homens. Verificou-se uma elevada prevalência isolada ou simultânea dos quatro comportamentos negativos relacionados à saúde entre os diabéticos.

Palavra-Chaves: Diabetes Mellitus. Tabagismo. Dieta para diabéticos. Abuso de álcool. Atividade motora.

\begin{abstract}
The aim of the present study was to determine the prevalence and simultaneity of negative behaviors related to health among individuals with diabetes according to sex and economic class. It is a crosssectional study in which were interviewed 337 individuals with diabetes in basic health units of Londrina, PR. Abusive consumption of alcohol, smoking, physical inactivity, and carelessness about diet were considered negative behaviors related to health. Among the interviewed diabetics, physical inactivity was present in $69.1 \%$, alcohol abuse in $21.1 \%$, negligent behavior on diet in $30.6 \%$ and smoking in $10.7 \%$. Differences in gender were observed in inactivity behavior $(p=0.008)$, which was greater among women, and smoking $(p<0.001)$ and abusive consumption of alcohol $(p<0.001)$, greater among men. Differences between economic classes and behaviors were not verified. The simultaneity of negative behaviors related to health consisted of the predominance of the sum of 2 negative behaviors, and the accumulation of negative behaviors was more prevalent among men. There was a high isolated or concurrent prevalence of the four health related behaviors among diabetics.
\end{abstract}

Keywords: Diabetes Mellitus. Smoking. Diabetic diet. Alcoholism. Motor activity

\footnotetext{
${ }^{1}$ Doutoranda em Saúde Coletiva na Universidade Estadual de Londrina. Email: mauiruska@gmail.com

${ }^{2}$ Doutor em Saúde Coletiva. Professor Adjunto da Universidade Estadual de Londrina.

${ }^{3}$ Doutor em Saúde Pública. Professor Adjunto da Universidade Estadual de Londrina.

${ }^{4}$ Doutor em Enfermagem. Professor Adjunto da Universidade Estadual de Londrina.

${ }^{5}$ Doutor em Saúde Coletiva. Professor Adjunto da Universidade Estadual de Londrina.
} 


\section{Introdução}

O Diabetes Melitus (DM) é um problema de saúde pública, tanto por suas altas taxas de morbimortalidade como pelas repercussões sociais e econômicas (SOLLA et al., 2004). É um indicador macroeconômico, uma vez que cresce rapidamente em países pobres e em desenvolvimento, atingindo pessoas em plena vida produtiva, onerando a previdência social e contribuindo para a continuidade do ciclo vicioso da pobreza e da exclusão social (BRASIL, 2006).

A prevalência desta doença vem aumentando exponencialmente em vários países, particularmente naqueles em desenvolvimento, e prevê-se que, em 2030, 366 milhões de indivíduos sejam portadores de DM, dos quais dois terços habitarão países em desenvolvimento (WORLD HEALTH ORGANIZATION, 2010). No Brasil, em 2000, havia aproximadamente4,5milhõesdeindivíduosportadores de DM (WORLD HEALTH ORGANIZATION, 2010), dos quais 50\% desconheciam seu diagnóstico (INTERNATIONAL DIABETES FEDERATION, 2006), com previsão de que em 2030 essa doença atinja 11 milhões de portadores da doença (WORLD HEALTH ORGANIZATION, 2010). Estudo multicêntrico, realizado entre 1986 e 1988 em nove capitais brasileiras com população na faixa etária entre 30 e 69 anos, encontrou uma prevalência de diabetes mellitus de 7,6\% (MALERBI; FRANCO, 1992). Outro estudo de base populacional, realizado em Pelotas (RS), no ano de 2000, obteve prevalência semelhante: 7,1\% entre 1.968 indivíduos de 20 a 69 anos estudados (COSTA et al., 2006). Entretanto, outros estudos têm apresentado prevalências maiores. Entre esses, destaca-se um estudo realizado em Ribeirão Preto (SP), com amostra de 1.473 indivíduos com idade entre 30 e 69 anos, que encontrou uma prevalência de DM de 12,1\% (TORQUATO et al., 2003). O inquérito realizado pelo Ministério da Saúde em 15 capitais brasileiras observou prevalências variando de $8 \%$ (Aracajú - SE) a 15\% (Manaus - AM) de DM em pessoas de 40 anos ou mais (BRASIL, 2003).
Este aumento na prevalência de DM é resultado de múltiplos fatores, tais como as mudanças socioculturais induzidas pela industrialização, o consumo de dietas hipercalóricas, a diminuição de práticas de atividade física, o aumento da obesidade, as mudanças no estilo de vida tradicional, e pelo aumento da expectativa de vida da população (AMERICAN DIABETES ASSOCIATION, 2007; OLIVEIRA et al., 2009).

A prevalência do diabetes, as projeções para o futuro e os fatores associados ao seu crescimento levam a uma justificada e crescente preocupação com os custos decorrentes deste panorama. Mundialmente, os custos diretos para atendimento aos portadores de DM oscilam de 2,5\% a 15\% dos gastos em saúde, variação decorrente de diferenças na prevalência da doença e na complexidade do tratamento disponível em cada local. Portadores de diabetes necessitam de duas a três vezes mais recursos para o cuidado com a saúde do que aqueles que não apresentam a doença (BARCELÓ et al., 2003). Muitos desses gastos, assim como a diminuição da qualidade de vida dos portadores de DM poderiam e podem ser prevenidos. Nesse sentido a adoção de cuidados adequados se torna fundamental para a prevenção de complicações do DM, as quais se constituem fatores que comprometem a qualidade de vida dos portadores da doença como o aumento do risco de mortalidade. Nessa discussão os serviços de atenção básica, por meio da atuação das equipes do Programa de Saúde da Família (PSF) (BRASIL, 2005), desempenham papel relevante para a prevenção de complicações em portadores de DM.

Encontra-se entre as ações preventivas de complicações do DM; a prática de atividade física, a realização de dieta adequada, a diminuição de ingestão de bebidas alcoólicas e o combate ao tabagismo, que, no acompanhamento do indivíduo com diabetes, em sua maior parte, fazem parte do seu tratamento, mas que representam para os serviços de saúde um grande desafio. Dessa forma, uma melhor compreensão sobre a prevalência e a 
inter-relação entre estes hábitos pode auxiliar na proposição de estratégias que sejam efetivas, no sentido de aumentar as chances de sujeitos com diabetes a adotarem comportamentos considerados saudáveis. Sendo assim, o objetivo deste trabalho foi investigar a prevalência de determinados comportamentos negativos relacionados à saúde, segundo sexo e classe econômica, em indivíduos com diabetes cadastrados e acompanhados por equipes de Saúde da Família.

\section{Material e Métodos}

Este é um estudo transversal e foi desenvolvido na zona urbana do município de Londrina-PR, em duas unidades básicas de saúde (UBS) da região central do município. Antes da escolha das UBS, foram solicitados à Diretoria de Ações em Saúde do município dados referentes ao número de pacientes cadastrados no programa de acompanhamento e cadastramento de diabéticos e hipertensos. Foram escolhidas as duas UBS com o maior número de diabéticos cadastrados, totalizando 367 sujeitos.

Nas duas unidades escolhidas os critérios de inclusão para a participação deste estudo foram: ser portador de DM do tipo 2, ser cadastrado e acompanhado em uma das unidades básicas de saúde participantes do estudo, ser residente no município de estudo e apresentar capacidade de raciocínio conservada no momento da entrevista.

Para a coleta de dados foi utilizado um formulário elaborado pelos pesquisadores, que foi previamente avaliado por professores doutores com experiência na área de saúde coletiva. Além disso, o instrumento foi previamente testado na realização de entrevistas em uma UBS que não foi incluída no estudo. Este teste piloto resultou na modificação de algumas questões do formulário, que melhor se adaptou ao perfil e a realidade dos entrevistados.

Entre as variáveis incluídas no formulário estavam; sexo (feminino/masculino), idade (em anos), classificação econômica da ABEP (categorizado em classes: A, B, C, D E e posteriormente recategorizado em A-B e C-D-E) (ASSOCIAÇÃO BRASILEIRA DE EMPRESAS DE PESQUISA, 2008), escolaridade (anos), situação conjugal (com e sem parceiro), tempo de diagnóstico de diabetes (em anos e posteriormente categorizado em 10 anos), tipo de tratamento do diabetes (nenhum, medicamento oral e insulina), presença de hipertensão (sim/não), presença de complicações do diabetes (sim/não) e dieta para o controle do diabetes (sim/não).

As variáveis dependentes do estudo (comportamentos negativos relacionados à saúde) tiveram como referencia temporal a realização no momento da entrevista e seguiram os seguintes critérios: foram considerados insuficientemente ativos no lazer os sujeitos que não acumulavam, no mínimo, 150 minutos semanais de prática de atividade física no tempo livre (ILANNEPARIKKA, 2010). Foram classificados como tabagistas os sujeitos que mencionaram fumar atualmente, independentemente da quantidade. O consumo abusivo de álcool foi definido considerando-se a ingestão de mais de quatro (mulheres) ou cinco (homens) doses de bebidas alcoólicas em uma mesma ocasião nos últimos 30 dias (BRASIL, 2011). A realização de dieta foi avaliada por meio da seguinte pergunta: o(a) $\operatorname{Sr}($ a) realiza algum cuidado na dieta para o controle do diabetes? Posteriormente para análise de simultaneidade foi considerado o somatório dos comportamentos apresentados.

As entrevistas foram realizadas no último semestre de 2009, e só foram entrevistados os indivíduos que concordaram em participar e assinaram o termo de consentimento livre e esclarecido. Os procedimentos metodológicos foram aprovados pelo Comitê de Ética em Pesquisa com Seres Humanos da Universidade Estadual de Londrina (Parecer CAAE 0206.0.268.000-08).

Os dados obtidos foram duplamente digitados em banco de dados criado no programa Epi Info, 
versão 3.5.3 para Windows ${ }^{\circledR}$. Os dois arquivos criados foram comparados pelo mesmo programa, e os dados discrepantes $(0,7 \%$ dos campos) foram corrigidos, para, em seguida, obter-se o banco de dados final para análise. O teste de Shapiro-Wilk foi utilizado para verificar a normalidade dos dados. Constatada a não normalidade na maioria das variáveis, optou-se pelo teste Qui-quadrado para a avaliação das possíveis associações.

\section{Resultados}

Dos 367 sujeitos amostrados, 30 deles (8,2\%) recusaram participar do estudo. Entre os 337 diabéticos entrevistados a idade variou entre 30 e 91 anos (mediana=65 anos). Na analise das características demográficas e clínicas observouse que a maior parte da amostra apresentou idade superior a 56 anos $(78,2 \%)$, classe econômica C-E $(86,8 \%)$ e escolaridade inferior a oito anos $(73,0 \%)$, situação conjugal com companheiro $(63, \%)$, com tempo de diagnóstico de diabetes menor que 10 anos (52,6\%), realizava controle do diabetes com uso de hipoglicemiantes $(70,6 \%)$, tinham elevadas prevalências de doença cardiovascular $(14,4 \%)$ e de hipertensão arterial $(69,1 \%)$ (dados não informados em tabela).

Conforme a Tabela 1, quanto aos comportamentos negativos relacionados à saúde, $233(69,1 \%)$ indivíduos relataram ter nível insuficiente de atividade física no lazer, $71(21,1 \%)$ consumir álcool abusivamente nos últimos 30 dias, 103 (30,6\%) não realizar dieta e $36(10,7 \%)$ ser fumantes. Comparando-se as prevalências dos comportamentos investigados entre os sexos, observou-se maior prevalência de inatividade física no lazer entre as mulheres. Por outro lado, o tabagismo e o consumo abusivo de álcool foram mais prevalentes entre os homens. Com relação à realização de dieta, não foi verificada diferença significativa na prevalência de homens e mulheres diabéticos investigados.

Tabela 1. Prevalência dos hábitos de vida segundo o sexo, entre portadores de diabetes em equipes de saúde da família, Londrina-PR, 2009.

\begin{tabular}{|l|c|c|c|c|c|c|}
\hline Variáveis & & & \multicolumn{3}{|c|}{ Classe econômica } \\
\hline & & & \multicolumn{2}{|c|}{ A-B } & \multicolumn{2}{|c|}{ C-D-E } \\
\hline & Total & $\%$ & n & $\%$ & n & $\%$ \\
\hline Inatividade Física no Lazer & & & & & & \\
\hline Sim & 233 & 69,1 & 25 & 56,8 & 208 & 71,0 \\
\hline Não & 104 & 30,9 & 19 & 43,2 & 85 & 29,0 \\
\hline Fumo & & & & & & \\
\hline Fumante & 36 & 10,7 & 6 & 13,6 & 30 & 10,2 \\
\hline Não fumante & 301 & 89,3 & 38 & 86,4 & 263 & 89,8 \\
\hline Dieta & & & & & & \\
\hline Não realizam & 103 & 30,6 & 13 & 29,5 & 90 & 30,7 \\
\hline Realizam & 234 & 69,4 & 31 & 70,5 & 203 & 69,3 \\
\hline Consumo abusivo de álcool & & & & & & \\
\hline Sim & & & & & & \\
\hline Não & & & & & & \\
\hline
\end{tabular}

Fonte: Autor. 
Quanto à classe econômica e os hábitos de vida investigados, apresentados na Tabela 2, não foram observadas diferenças significativas. De qualquermodo, é importante mencionar a elevada diferença numérica em relação à inatividade física no lazer e no consumo abusivo de álcool, e que os valores foram marginais ao $\mathrm{p}$-valor de significância adotado $(\mathrm{p} \leq 0.05)$. No caso, numericamente se observou maior prevalência de inatividade física no lazer entre os sujeitos com menor nível econômico, ao passo que a maior prevalência de consumo abusivo de álcool foi superior entre os sujeitos das classes econômicas mais elevadas.

Tabela 2. Prevalência dos hábitos de vida segundo classe econômica da Associação Brasileira de Empresas de Pesquisa (ABEP) entre portadores de diabetes em equipes de saúde da família, Londrina-PR, 2009.

\begin{tabular}{|l|c|c|c|c|c|c|}
\hline Variáveis & & & \multicolumn{3}{|c|}{ Classe econômica } \\
\hline & & & \multicolumn{2}{|c|}{ A-B } & \multicolumn{2}{|c|}{ C-D-E } \\
\hline & Total & $\%$ & $\mathrm{n}$ & $\%$ & $\mathrm{n}$ & $\%$ \\
\hline Inatividade Física no Lazer & & & & & & \\
\hline Sim & 233 & 69,1 & 25 & 56,8 & 208 & 71,0 \\
\hline Não & 104 & 30,9 & 19 & 43,2 & 85 & 29,0 \\
\hline Fumo & & & & & & \\
\hline Fumante & 36 & 10,7 & 6 & 13,6 & 30 & 10,2 \\
\hline Não fumante & 301 & 89,3 & 38 & 86,4 & 263 & 89,8 \\
\hline Dieta & 71 & 21,1 & 14 & 31,8 & 57 & 19,5 \\
\hline Não realizam & 103 & 30,6 & 13 & 29,5 & 90 & 30,7 \\
\hline Realizam & 234 & 69,4 & 31 & 70,5 & 203 & 69,3 \\
\hline Consumo abusivo de álcool & 78,9 & 30 & 68,2 & 236 & 80,5 \\
\hline Sim & & & & & & \\
\hline Não & & & & & & \\
\hline
\end{tabular}

Fonte: Autor.

figura 1 apresenta a análise simultânea dos comportamentos relacionados à saúde nos sujeitos investigados, segundo sexo. Observouse que a maior parte dos sujeitos apresentou pelo menos um dos quatro comportamentos negativos relacionados à saúde investigados: $82,2 \%$ (77,6\% das mulheres e $89 \%$ dos homens). Além do mais, houve diferença significativa entre os sexos, segundo número de comportamentos negativos. Entre as mulheres, $67,7 \%$ referiram até um comportamento negativo relacionado à saúde, ao passo que exatamente metade dos homens tinha esta mesma característica (até um comportamento negativo). 
Figura 1 - Frequência de simultaneidade de comportamentos negativos relacionados à saúde, segundo sexo em equipes de saúde da família, Londrina-PR, 2009.

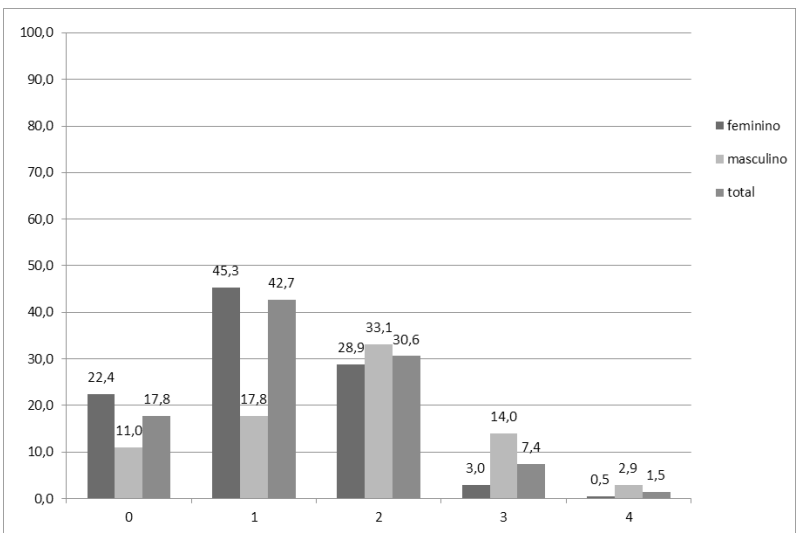

Fonte: Autor. * diferença significativa entre os sexos com p-valor $<0,001$.

\section{Discussão}

O presente trabalho investigou a prevalência de determinados comportamentos relacionados à saúde em diabéticos acompanhados por equipes de saúde da família. Em geral, os dados mostraram elevada prevalência dos quatro comportamentos negativos relacionados à saúde investigados (inatividade física no lazer, não realização de dieta, tabagismo e consumo abusivo de álcool), havendo algumas importantes diferenças entre os sexos.

Dos comportamentos investigados, a inatividade física no lazer foi o que apresentou maior prevalência. A proporção de diabéticos que não praticavam semanalmente, no mínimo, 150 minutos de atividade física no tempo livre foi de $69,1 \%$. Maior prevalência foi encontrada entre as mulheres. Essa elevada prevalência encontrada de prática insuficiente de atividade física no lazer é condizente com a literatura. Sabe-se que é alta a prevalência de inatividade física na população brasileira. Em estudo transversal realizado com diabéticos na atenção primária em Pelotas, RS (ARAÚJO et al., 1999), observou-se a não realização de atividade física em $79,1 \%$ da população estudada, achado muito semelhante ao do presente estudo. Um estudo prévio realizado em serviços da atenção básica demonstrou gasto aumentado (serviços médicos e de medicamentos) no atendimento de portadores de diabetes insuficientemente ativos fisicamente em comparação aos ativos, reforçando a importância da realização de atividade física no controle da doença (CODONGO; FERNANDES; MONTEIRO, 2012). Dados mais recentes do sistema de Vigilância de Doenças Crônicas (VIGITEL), por estudo realizado em inquérito telefônico (BRASIL, 2011), do Ministério da Saúde do Brasil, apontam que a inatividade física no lazer é, entre os diversos indicadores comportamentais investigados, o mais prevalente na população das capitais de estado brasileiros. Dados coletados em 2010 demonstram que a proporção de sujeitos insuficientemente ativos no lazer variou de 77,6\% (no Distrito Federal-DF) a 88,7\% (em Rio Branco- AC), ou seja, mais elevada do que os encontrados no presente estudo. Vale mencionar que, apesar das diferenças metodológicas e de população de ambos os estudos, a comparação se faz interessante, no sentido de que reforça o fato de ser elevada a proporção de sujeitos que não pratica atividade física em níveis adequados para influenciar positivamente a saúde.

Quanto à não realização de dieta, em torno de $30 \%$ dos sujeitos preferiu não realizar nenhum tipo de dieta especial para o controle da diabetes, não havendo diferença entre os sexos e os níveis econômicos. Dias et al. (2010) demonstraram o efeito positivo no controle dos níveis glicêmicos com a realização de dieta entre diabéticos. Por sua vez, Boas et al. (2011) avaliaram a adesão de pessoas com diabetes tipo 2 à dieta e ao exercício físico e também constataram baixa adesão. Peres; Franco e Santos (2006), investigando o comportamento alimentar de mulheres diabéticas atendidas por uma unidade básica de saúde, observaram que muitas referem dificuldade para seguir a dieta prescrita, inclusive em função de referirem perda do prazer de comer e da autonomia para se alimentar. Isso faz com que o comportamento alimentar, assim como 
as outras variáveis comportamentais, tenha que ser compreendido a partir não apenas do referencial biológico, mas também em seus aspectos sociais, culturais, psicológicos e econômicos.

Em relação ao tabagismo, verificou-se prevalência de $10,7 \%$, com maior prevalência entre os homens, resultado este semelhante aos encontrado por Cabrera de Leon et al. (2009), que, investigando uma população espanhola, observaram maior prevalência de fumantes entre os homens, sendo esta diferença maior entre homens e mulheres diabéticas do que na população em geral. Comparando nossos achados com os do VIGITEL (BRASIL, 2011), observaram-se, no total, valores próximos à prevalência mais baixa encontrada entre as capitais brasileiras investigadas, no caso, 8,3\% em Salvador-BA.

Quanto ao consumo abusivo de álcool, observou-se prevalência elevada, especialmente entre os homens. Ao contrário do fumo, em que a prevalência total encontrada neste estudo esteve próxima à da capital de estado brasileiro com menor prevalência, no consumo abusivo de álcool, a prevalência do álcool $(21,1 \%)$ esteve mais próxima da capital com maior proporção de consumidores abusivos (25,2\%; Recife-PE). Esta aproximação se deveu mais aos homens, já que, entre estes, $35,3 \%$ dos sujeitos pesquisados referiram consumo abusivo nos últimos 30 dias (no caso do VIGITEL, a prevalência mais elevada foi encontrada em Natal$\mathrm{RN}-37,1 \%$ ). No caso das mulheres, a prevalência observada no presente estudo $(11,4 \%)$ ficou em uma situação intermediária, entre a mais baixa $(5,6 \% \mathrm{em}$ Manaus-AM) e a mais alta (17,3\% em Salvador-BA) das prevalências encontradas nas capitais brasileiras (BRASIL, 2011).

A análise de simultaneidade demonstrou que é baixa a proporção de sujeitos sem nenhum comportamento negativo relacionado à saúde. A maior parte apresenta pelo menos um comportamento negativo, sendo esta proporção maior entre os homens.

\section{Conclusões}

As altas prevalências de comportamentos negativos relacionados à saúde, seja isoladamente ou conjunta, observadas no presente estudo reforçam a idéia de uma abordagem preventiva que enfoque o enfrentamento desses de maneira simultânea. É evidente que cada comportamento apresenta suas especificidades, mas, como o Sistema Único de Saúde tem como um de seus princípios a integralidade, parece contraditório focar cada comportamento de maneira isolada, inclusive porque muitas vezes a mudança de um determinado comportamento (por exemplo: a prática de atividade física) pode ser um importante estímulo e facilitador para que outros comportamentos (adotar uma alimentação mais saudável e parar de fumar, por exemplo) sejam adotados.

De qualquer modo, há de se destacar que a atuação sobre os comportamentos relacionados à saúde da população não é tarefa fácil. Como já foi dito anteriormente, há de se considerar aspectos culturais, sociais, econômicos, entre outros, ou seja, faz-se necessário compreender a complexidade do comportamento humano, inclusive para se fugir de práticas meramente prescritivas e que, muitas vezes, tratam o comportamento humano como uma questão de ordem moral. Hallal e Florindo (2011) lembram que não é função da saúde pública controlar o comportamento das pessoas, mas, sim, buscar promover condições para que as pessoas possam realizar escolhas saudáveis.

Tudo isso é importante tanto para a prevenção como para o controle de algumas doenças, ainda mais no caso de pessoas com diabetes, para quem o tratamento não medicamentoso, baseado essencialmente em mudanças comportamentais, é fundamental. Neste sentido, o Sistema Único de Saúde brasileiro tem avançado nos últimos anos, estando atento às mudanças epidemiológicas e sociais pelas quais vem passando o Brasil, contudo o tratamento das doenças crônicas não transmissíveis ainda se configura um grande desafio (PAIM et al., 
2011). Nossos dados reforçam esta idéia, no sentido de que faz se necessário que a atenção primária busque atuar sobre os comportamentos relacionados à saúde, de modo a melhorar a saúde das pessoas com diabetes, diminuindo a incidência deste agravo.

\section{Agradecimentos}

Agradecemos ao apoio da Coordenação de Aperfeiçoamento de Pessoal de Nível Superior (CAPES), pela bolsa de estudos, os serviços da atenção básica de Londrina que possibilitaram e apoiaram a realização deste estudo, bem como aos portadores de diabetes que aceitaram fazer parte do mesmo.

\section{Referências}

AMERICAN DIABETES ASSOCIATION. Nutricion recommendations and interventions for Diabetes: A position statement of the American Diabetes Association. Diabetes Care, Indianapolis, v.30, Suppl 1, p.48-65, 2007.

ARAÚJO, R.B.; SANTO,I;CAVALETI,M.A.; COSTA, J.S.D.; BÉRIA, J.U. Avaliação do cuidado prestado à pacientes diabéticos em nível primário. Revista Saúde Pública. São Paulo, v.33, n.1, p.24-32, 1999.

ASSOCIAÇÃO BRASILEIRA DE EMPRESAS DE PESQUISA. CCEB 2008 - Critério de classificação econômica Brasil. Disponível em: <http://www.abep. org/codigosguias/Criterio_Brasil_2008.pdf $>$. Acesso em: 11 out. 2008.

BARCELÓ, A.; AEDO, C.; RAJPATHAK, S.; ROBLES, S. The cost of diabetes in Latin America and the Caribbean. Bulletin of the World Health Organization, Genebra, v.81, n.1, p.19-26, 2003.

BOAS, L.C.G.; FOSS, M.C.; FOSS-FREITAS, M.C.; TORRES, H.C.; MONTEIRO, L.Z.; PACE, A.E.Adesão à dieta e ao exercício físico das pessoas com diabetes mellitus. Texto \& contexto Enfermagem. Florianópolis, v.20, n.2, p.272-279, 2011.
BRASIL. Ministério da Saúde. Secretaria de Atenção à Saúde. Departamento de Atenção Básica. Diabetes mellitus. Brasília: Ministério da Saúde, 2006.

BRASIL. Ministério da Saúde. Secretaria de Atenção à Saúde. Departamento de Atenção Básica. Saúde da Família. Brasília: Ministério da Saúde, 2005.

BRASIL. Ministério da Saúde. Secretaria de Vigilância em saúde. Instituto Nacional de Câncer. Inquérito domiciliar sobre comportamentos de risco e morbidade referida de doenças e agravos não transmissiveis. Brasília: Ministério da Saúde, 2003.

BRASIL. Ministério da Saúde. Vigilância de fatores de risco e proteção para doenças crônicas por inquérito telefônico (VIGITEL). Brasília; Ministério da Saúde, 2011.

CABRERADELEÓN,A.; CASTILHO RODRÍGUEZ, J.C.; DOMÍNGUEZ, C.S.; RODRÍGUEZ PÉREZ, M.C.; BRITO DÍAZ, B.; ÁLAMO, A.B.; FERNANDÉZ, L.C.; GONZÁLEZ, D.A.; SÁNCHEZ, J.J.A.; HERNANDÉZ, A.G.; AGUIRRE-JAIME, A. Estilo de vida y adherencia al tratamiento de la población canaria con diabetes mellitus tipo 2. Revista Española de Salud Publica. Madri, v.83, n.4, p.567-575, 2009.

CODOGNO, J.S.; FERNANDES, R.A.; MONTEIRO, H.L. Prática de atividades físicas e custo do tratamento ambulatorial de diabéticos tipo 2 atendidos em unidade básica de saúde. Arquivos Brasileiros de Endocrinologia \& Metabologia.São Paulo, v.56, n.1. p.6-11, 2012.

COSTA, J. S. D.; OLINTO, M.T.A.; ASSUNÇÃO, M.O.F.; GIGANTE, D.P.; MACEDO, S.; MENEZES, A.M.B. Prevalência de Diabetes Mellitus em Pelotas, RS: um estudo de base populacional. Revista de Saúde Pública, São Paulo, v. 40, n. 3, p.542-5, jun. 2006.

DIAS, V.M.; PANDINI, .JA.; NUNES, R.R.; SPERANDEL. S.L.M.; PORTELLA, E.S.; COBAS, R.A.; GOMES, M.B. Influência do índice glicêmico da dieta sobre parâmetros antropométricos e bioquímicos em pacientes com diabetes tipo 1. Arquivos Brasileiros de Endocrinologia \& Metabologia..São Paulo, v.54, n. 9, p.801-806, 2010. 
FLORINDO, A.A.; HALLAL, P.C. Um olhar para o futuro da epidemiologia da atividade física. In: FLORINDO, A.A.; HALLAL, P.C. Epidemiologia da atividade fisica. São Paulo: Atheneu; 2011.p.

ILANNE-PARIKKA， P.; LAAKSONEN， D. E.; ERIKSSON, J. G.; LAKKA, T. A.; LINDSTROM, J.; PELTONEN, M.; AUNOLA, S.; KEINANENKIUKAANNIEMI, S.; UUSITUPA, M.; TUOMILEHTO, J. Leisure-Time Physical Activity and the Metabolic Syndrome in the Finnish Diabetes Prevention Study. Diabetes Care. Indianapolis, v.33, n.7, p.1610-17, 2010.

INTERNATIONAL DIABETES FEDERATION. Diabetes Atlas. 3. ed. Brussel, 2006. Disponível em: $<$ http:// www.eatlas.idf.org/webdata/docs/background openingpc. pdf $>$. Acesso em: 10 jun. 2009.

MALERBI, D.; FRANCO, L. J. Multicenter study of the prevalence of diabetes mellitus and impaired glucose tolerance in the urban Brazilian population aged 30-69 yr. Diabetes Care, Indianápolis, v.15, n.11, p.1509-1516, 1992.

OLIVEIRA，A. F.; VALENTE， J.G.; LEITE， I.C.; SCHRAMM, J.M.A.; AZEVEDO, A.S.R.; GADELHA, A.M.J. Global burden of disease attributable to diabetes mellitus in Brazil. Caderno de Saúde Pública, Rio de Janeiro, v.25, n.6, p.1234-44, 2009.

PAIM, J.; TRAVASSOS, C.; ALMEIDA, C.; BAHIA, L.; MACINKO, J. The Brazilian health system: history, advances, and challenges. Lancet, v.9797, n.377, p.177897, 2011.

PÉRES, D.S.; FRANCO, L.J.; SANTOS, MA.. Comportamento alimentar em mulheres portadoras de diabetes tipo 2. Revista de Saúde Pública. São Paulo, v.40, n.2, p.310-7, 2006.

SOLLA, J. J. S. P.; FRANCO, L.J.; CAMPOS, G.P.; MACHADO, C.A.; LESSA, I. Entrevista: O enfoque das políticas do SUS para a promoção da saúde e prevenção das DCNT: do passado ao futuro. Ciência \& Saúde Coletiva, Rio de Janeiro, v.9, n.4, p.945-56, 2004.

WORLD HEALTH ORGANIZATION. Diabetes Programme. Disponível em: <www.who.int/diabetes/facts/ world_figures/em>. Acesso em: 10 fev. 2010.
Recebido em: 27 jun. 2013.

Aceito em: 3 jun. 2014. 
